\title{
Der Verlauf von Klatschgesprächen
}

\author{
Angela Keppler \\ Sozialwissenschaftliche Fakultät, Fachgruppe Soziologie, Universität Konstanz, Universitätsstraße 10, \\ D-7750 Konstanz 1
}

Zusammenfassung: Betrachtet man Klatschgespräche in ihrem zeitlichen Verlauf, so findet man ein Muster der sequentiellen Organisation, in dem sich diese kommunikative Gattung realisiert und reproduziert. In einer der eigentlichen Klatschmitteilung vorgeschalteten Klatschpräsequenz wird die Bereitschaft zum Klatschen intersubjektiv ausgehandelt und ratifiziert. In der nachfolgenden Klatschgeschichte wird eine auffällige, dem Zuhörer noch nicht bekannte Handlung eines abwesenden Dritten berichtet und bewertet, und zwar so, daß dem Handelnden ein bestimmter Charakter zugeschrieben wird. In der sich anschließenden Klatschnachsequenz wird die individuelle Charakterisierung im Blick auf soziale Typen generalisiert und in einem Meinungsaustausch erläutert. Interpretationen und Deutungsangebote werden gemacht, die die individuelle Handlung in den Rahmen allgemeiner sozialer und moralischer Regeln stellen.

Auf der Straße von Phaleron nach Athen langweilt sich ein Mensch; da bemerkt er einen anderen, der vor ihm dahinwandert, holt ihn ein und bittet ihn um einen Bericht über das Gastmahl, das Agathon gegeben hat. So entsteht die Theorie der Liebe: aus Zufall, Langeweile, aus Lust an der Unterhaltung oder, wenn man das vorzieht, aus einem drei Kilometer langen Klatsch. Aristodemos hat am berühmten Gastmahl teilgenommen; er hat davon Apolodorus erzählt, der es auf der Straße von Phaleron Glaukon weitergibt (wie man sagt ein Mensch ohne philosophische Kultur) und es, durch das Medium des Buches, uns erzählt, die wir jetzt davon reden. Das Gastmahl ist also nicht einfach nur eine ,Unterhaltung* (wir sprechen über eine Frage), sondern auch Klatsch (wir sprechen unter- und miteinander über andere).

R. Barthes

„Unter- und miteinander über andere" - über abwesende andere - sprechen, das ist für den Anfang keine schlechte Bestimmung von Klatsch. Zureichend kann diese Definition nicht sein. Denn in Klatschgesprächen tauschen wir nicht etwa nur Informationen über die abwesenden anderen aus. Wäre es so einfach, stünde es anders um den Ruf dieser Form der Kommunikation. Als leeres, Geschwätz' und nichtiges ,Gerede', das einen Mangel an sozialer Verantwortlichkeit offenbart, als ein „Musterbeispiel für die gesellschaftliche Produktion sinnentleerten Geredes", wird das Reden über andere bis heute nicht selten verurteilt. ${ }^{1}$ Das Gerede dient auch nach Ansicht so mancher Philoso-

\footnotetext{
' So berichtet die Frankfurter Allgemeine Zeitung vom 17. 10. 1985 von einem Landtagsabgeordneten, der verkündet habe, daß sich „auch die Alltagspolitik endlich wirksam vor Klatsch, Tratsch und Indiskretionen schützen“ müsse.
}

phen nur zur Verhinderung von wirklichem Verstehen und Begreifen. Heidegger (1979 : 169) zum Beispiel schreibt: „Das Gerede ist die Möglichkeit, alles zu verstehen ohne vorgängige Zueignung der Sache. . . Das Gerede, das jeder aufraffen kann, entbindet nicht nur von der Aufgabe echten Verstehens, sondern bildet eine indifferente Verständlichkeit aus, der nichts mehr verschlossen ist." Unterstellt wird dem Reden über die Angelegenheiten anderer häufig die Verbreitung von Halbwissen, von Bosheiten, von Verleumdungen; im Reden über andere wird eine Bedrohung ihrer personalen Integrität gesehen. Diesen Aspekt führt auch R. Barthes (1985: 155) aus: „Der Klatsch reduziert den Anderen auf ihn/sie, und diese Reduktion ist für mich unerträglich. Der Andere ist für mich weder er noch sie; er hat nur seinen eigenen Namen, seinen Eigennamen. Das Pronomen der dritten Person ist ein bösartiges Pronomen: es ist das Pronomen der Unperson, es rückt fern, es erklärt für nichtig. "

Im Unterschied dazu heben soziologische, ethnologische und psychologische Untersuchungen zumeist die positiven Aspekte der Klatschkommunikation hervor. Klatsch, so die weitverbreitete These, ist ein Katalysator für soziale Prozesse. Er dient der Gruppenkontrolle und der emotionalen Stabilität. Durch ihn werden Informationen und soziale Wertvorstellungen in Umlauf gebracht, er hält die soziale Ordnung aufrecht und stärkt den Zusammenhalt von Gemeinschaften; er liefert einen Modus der Sozialisation und der sozialen Kontrolle, erleichtert Selbsterkenntnis und Selbsteinschätzung, indem er eine Basis für soziale Vergleiche abgibt, er hat eine kathartische Funktion und konstituiert eine Form der Wunscherfüllung, hilft Streitigkeiten zu kontrollieren, erleichtert die Aus- 
wahl von Führern und schafft Macht. Er gibt die Möglichkeit zur Selbstoffenbarung und zur Überprüfung ethischer Entscheidungen. (s. Gluckman 1963; Paine 1967; Harding 1975; Suls 1977; Sabini/ Silver 1982).

Die Verurteilung und das Lob des Klatsches haben eines gemeinsam: Sie beschäftigen sich nahezu ausschließlich mit seinen individual- und /oder sozialpsychologischen Folgen und Funktionen. Die konkrete Erscheinungsform der kommunikativen Gattung interessiert dabei nicht. Klatsch wird auf seine Inhalte reduziert und mit seinen vermeintlichen Wirkungen identifiziert. Sozial-Anthropologen oder Ethnologen, die die Rolle der Klatschkommunikation in und für bestimmte Kulturen untersuchen, beschreiben zumeist, was Leute über den Klatsch sagen, nur selten wird festgehalten, was $i m$ Klatsch gesagt wurde und Untersuchungen über die Art und Weise, wie geklatscht wird, haben den Seltenheitswert kostbarer Sammlerobjekte.

Die vorliegende Untersuchung hat sich demgegenüber das Ziel gesetzt, den Klatsch an Hand von Aufzeichnungen und Transkriptionen natürlicher Interaktionsabläufe zu analysieren. ${ }^{2}$

Dabei bleibt seine ursprüngliche Ereignisform in ihrem realen zeitlichen Ablauf erhalten. Die Analyse konzentriert sich auf diverse in privaten und öffentlichen Kontexten aufgezeichnete Gespräche, in deren Verlauf über Verhaltens- und Handlungsweisen anderer gesprochen wird. Am Ausgangspunkt der Untersuchung stehen die folgenden Fragen: Wie läßt sich der Klatsch als eine eigenständige Form mündlicher Kommunikation beschreiben? Wie lassen sich seine Strukturmerkmale analytisch bestimmen? Und nicht zuletzt: Wie unterscheidet sich die Klatschkommunikation von anderen Formen des Informationsaustauschs über abwesende Personen? Zur Vorbereitung der Antworten ist ein Blick auf die Ausgangssituation von Klatschgesprächen nützlich. Am Klatsch beteiligt

\footnotetext{
Diese Analyse wurde im Rahmen des von Thomas Luckmann und Jörg R. Bergmann geleiteten DFGForschungsprojekts "Rekonstruktive Gattungen der alltäglichen Kommunikation" unternommen. Den Diskussionen mit den Projektleitern und den Projektmitarbeitern (Bernd Ulmer/Hubert Knoblauch) verdankt sie wesentliche Anregungen. Eine extensive Bearbeitung des Themas bietet J.R. Bergmann in seiner im Sommer 1986 fertiggestellten Habilitationsschrift ,Klatsch als Gattung der alltäglichen Kommunikation. Zur Sozialform der diskreten Indisk retion“.
}

sind immer zumindest drei Parteien: ein aktiv Klatschender, der im Besitz ,klatschwürdiger‘ Informationen ist, ein passiv Klatschender, der ein Interesse an diesen Mitteilungen hat und schließlich, wenn auch indirekt, eine dritte - abwesende Person, deren Angelegenheiten die Informationen betreffen. Nehmen wir dieses Grundmuster der personalen Konstellation, das sich an der äußeren sozialen Erscheinungsform von Klatsch ablesen läßt ${ }^{3}$, als gegeben an, so stellen sich für eine Analyse des Interaktionsablaufs die weiteren Fragen: Wie teilt der aktiv Klatschende seinem Gegenüber mit, daß er im Besitz gewisser Informationen ist? Wie macht der passiv Klatschende sein Interesse an diesen Informationen deutlich, und nicht zuletzt: Wie werden diese Informationen übermittelt?

Die Rede vom Klatsch als einer spezifischen Gattung der mündlichen Kommunikation ist nur dann berechtigt, wenn gezeigt werden kann, daß es vom jeweiligen Kontext unabhängige Regelhaftigkeiten gibt, die, in Absehung von den je individuellen Besonderheiten einzelner Klatschgespräche, unter allen Umständen interaktiv realisiert werden. Dies bedeutet aber nicht, daß eine analytische Bestimmung allein der Strukturmerkmale ausreicht, um zu beschreiben, wie geklatscht wird. Die Art und Weise des Klatschens hängt immer auch von den je besonderen kontextuellen Bedingungen ab, die in einem zweiten Schritt der Analyse mit einbezogen werden müssen. Zunächst jedoch gilt es, den Blick auf das immer wiederkehrende Ablaufmuster zu konzentrieren, in dem sich diese kommunikative Gattung in der Abfolge von Klatschpräsequenz, Klatschgeschichte und Klatschnachsequenz interaktiv realisiert und reproduziert. Zunächst einmal können wir bei nahezu allen Klatschgesprächen eine einleitende Phase registrieren, in der ausgehandelt wird, ob ein Klatschgespräch folgen kann oder nicht. Hier wird zum einen das Klatschobjekt identifiziert und zum anderen die Situation als geeignet oder ungeeignet für ein Klatschgespräch definiert. Erst nach, erfolgreichem` Abschluß dieser einleitenden Phase folgt in einem mittleren Teil die Klatschgeschichte und in einer dritten Sequenz im Anschluß daran eine Kommentierung bzw. $\mathrm{Ge}$ neralisierung des singulären Ereignisses. Von der persönlichen, das Besondere hervorhebenden

\footnotetext{
${ }^{3}$ J. R. Bergmann (1985) führt in diesem Zusammenhang den Begriff der "Klatschtriade“, bestehend aus „Klatschobjekt“, „Klatschproduzent" und „Klatschrezipient", ein.
} 
Klatschmitteilung wird auf ein damit zusammenhängendes allgemeines Phänomen geschlossen; Interpretationen oder Deutungsangebote werden gemacht, die die individuelle Handlung in den Rahmen allgemeiner moralischer oder sozialer Normen stellen.

Eine Grobstruktur der sequentiellen Ordnung liest sich wie folgt:

Klatschpräsequenz: Intersubjektive Bereitschaft Klatschgeschichte: Individuelle Charakterisierung Klatschnachsequenz: Soziale Generalisierung

Jede dieser Sequenzen wird im folgenden an Hand einzelner Gesprächsausschnitte im Hinblick auf ihre konstitutiven Merkmale beschrieben und analysiert.

\section{Die Klatschpräsequenz}

Wie zu Beginn bereits deutlich wurde (und wie uns allen aus dem Alltag bestens bekannt ist), handelt es sich beim Klatschen um eine - unter sozialen und moralischen Gesichtspunkten betrachtet - als ,unerwünscht', ,unseriös' oder gar ,schädlich' stigmatisierte Aktivität, die sich nichtsdestotrotz groBer Beliebtheit erfreut. Die offizielle Diskriminierung von Klatsch, die diesem einen ambigen Charakter gibt, bleibt nicht ohne Folgen für die Pragmatik dieses Genres. Sie findet ihren Niederschlag in einer, der Klatschmitteilung vorangestellten, Klatschpräsequenz, die unabhängig von den jeweiligen Realisierungsbedingungen spezifische Merkmale aufweist. Folgen hat die öffentliche Ächtung vor allem für den Ablauf und die personale Konstellation, in der geklatscht wird. Die personale Konstellation, die Jörg Bergmann (1985:13) in der Formel „Geklatscht wird nur über Freunde und Bekannte und nur mit Freunden und Bekannten" zusammenfaßt, bedeutet eine Form der Absicherung und der eingeschränkten Verbreitung eines Wissens. Die soziale Ambiguität, die eine Absicherung dieses konversationellen Genres notwendig macht, spiegelt sich in seinem Ablauf, in der sequentiellen Folge wieder. Eine Klatschgeschichte wird erst dann erzählt, wenn in einer vorangehenden Gesprächssequenz die intersubjektive Bereitschaft aller am Gespräch Beteiligten zum Klatschen erkennbar wird. Je nach dem Intimitätsgrad der Beziehungen der Akteure untereinander fällt diese Sequenz lang oder kurz, detailliert oder summarisch aus. Hier kann aber, und das ist entscheidend, ein Angebot zum Klatschen indirekt gemacht und vom anderen ebenso indirekt abgelehnt werden, ohne daß eine offene Ablehung zu einer möglicherweise peinlichen Situation für alle Beteiligten führen müßte. Derartige Präsequenzen finden sich zum Beispiel auch bei Bitten, Nachfragen oder Ankündigungen; in all diesen Fällen dienen sie ebenso wie beim Klatsch der Vermeidung von „disprefered alternatives“" (s. Terasaki 1976). Auch beim Klatsch dient die Präsequenz der Vermeidung einer „unerwünschten Alternative“, die eine offene Weigerung zur Beteiligung am Klatsch, aufgrund der sozialen Ächtung mit der dieser belegt ist, darstellen würde. Eine offene Zurückweisung eines Klatsch-Angebots oder einer KlatschNachfrage wäre für alle Gesprächsteilnehmer unangenehm. In der Präsequenz werden deshalb Angebote diskret unterbreitet und Nachfragen eher implizit angedeutet als explizit gestellt. Damit bleiben dem Partner diverse Handlungsmöglichkeiten offen. Er bestimmt den Charakter, den das folgende Gespräch annimmt, mit. Die nachfolgenden Gesprächsausschnitte ${ }^{4}$ zeigen - in dieser Reihenfolge -

- wie eine Meinungsverschiedenheit zum Klatsch führt (Beispiel 1 u. 2)

- wie ein Klatschangebot akzeptiert wird (Beispiel 3)

- wie eine Klatschnachfrage zum Erfolg führt (Beispiel 4)

- wie ein potentielles Klatschgespräch scheitert (Beispiel 5)

${ }^{4}$ Das Transkriptionssystem basiert weitgehend auf dem von Gail Jefferson für die amerikanische Konversationsanalyse entwickelten System.

I Beginn einer Überlappung, d.h. gleichzeitiges Sprechen von zwei Personen

] Ende einer Überlappung

$=$ Schneller AnschluB

$(0,5)$ Pause; Dauer in Sekunden

(-) Kurze Pause (ca. 0,25 Sekunden)

: Dehnung

${ }^{\circ} \mathrm{ja}^{\circ} \quad$ Leise gesprochen

nein Betont gesprochen

NEIN Laut gesprochen

Satzzeichen indizieren nur Intonationsvorgänge:

,? schwach bzw. stark steigende Intonationskurve

; . schwach bzw. stark sinkende Intonationskurve

ohh Hörbares Einatmen

hh Hörbares Ausatmen

( ) Inhalt der Äußerung unverständlich

(( )) Kommentar des Transkribienten Auslassung im Transkript

Zum Schutz der beteiligten Personen werden alle vorkommenden Personennamen sowie Orts- und Zeitangaben durch Pseudonyme ersetzt. 


\section{Beispiel 1:}

AK : PM 13B/84: 3 „Nichts Tolles“

09 Monika: Un der Metzger der hat ne Profes-

11 Erich: sur in X-Stadt;

12 Monika:

13

14

15 Erich:

16

17 Monika: Nich?

18 Erich: ${ }^{\circ}$ Find ich nich also, ${ }^{\circ}$

19 Monika: Hmhh?

20 Erich: Vielleicht war er ganz nett!

21 Monika: Ja ich kenn den nich näher nur so 22

23

24

25

26 Monika: Abr du kanntest ihn besser odr wie?

Beispiel 2:
03

04

09 Herr G.: Die habn mich gemahnt; die habn mich

In diesen verschiedenen Klatschpräsequenzen findet zuallererst eine wechselseitige Identifikation des zukünftigen Klatschobjekts - der abwesenden Person, über die Näheres erzählt werden soll statt. Diese wird hier, ganz so wie Roland Barthes es als unerträglich beschreibt, auf "ihn“ bzw. ,sie“" reduziert. Denn nachdem eine Person durch die Nennung ihres Vor- und/oder Nachnamens in der ersten Äußerung ins Gespräch eingeführt wird, nimmt der Rezipient in der zweiten Äußerung auf diese durch einen Rekurs in pronominalisierter Form Bezug. Auf weitere Angaben zur personalen Identifikation wird zumeist verzichtet, der erste Sprecher setzt voraus, daß sein Gesprächspartner die betreffende Person, sowie gewisse sie betreffende Umstände kennt. Der zweite Sprecher bestätigt dies entweder explizit (s. Beispiel 1), oder/ und durch einen Rekurs in pronominalisierter Form (s. Beispiel 2; 3; 4), oder implizit, indem der Angesprochene gleich Informationen über sie weitergibt (s. Beispiel 5). Die Art und Weise, wie die abwesende Person ins Gespräch eingeführt wird, macht in allen Fällen deutlich, daß es sich um eine/ n gemeinsame/n Bekannte/n handelt. Bei der Identifikation wird auf ein gemeinsames Vorwissen der Interaktionspartner Bezug genommen, das zum einen die abwesende Person, zum anderen aber darüber hinaus auch ganz spezifische Umstände betreffen kann. ${ }^{5}$ Diese einleitenden Bemerkungen zeigen, daß die Gesprächsteilnehmer nicht nur ein gemeinsames Wissen über die abwesende Person haben, sie demonstrieren auch den spezifischen Charakter der Relation, die zwischen den Interagierenden besteht. Eine Relation, in der soziale Distanz minimiert ist. Die Verständigung darüber, daß man über gemeinsame Bekannte spricht, erfolgt aber nicht in einem quasi neutralen Zusammenhang, sondern sie ist Teil einer durch evaluative Komponenten geprägten Eröffnungsphase: Klatsch wird durch wertende Äußerungen vorbe-

\footnotetext{
5 Beim Gesprächssegment „Sizilianisch“ wird eben vorausgesetzt, daB alle über die Tatsache, daß Tante Berta nach Sizilien geflogen ist, Bescheid wissen. Beim Gespräch „Ganz umgänglich“ wird vorausgesetzt, daß Sonja weiß, welche Situation Marlies anspricht.
}

klappt das jetzt noch in dem Seme-
AK : EM 28A/84 : 8 „Promotion“
01 Herr R.: Wie is n das jetz eigntlich mit der
02
Promotion von dem Ketteler (-)
AK : PM 14B/84 : 4 „Sizilianisch“

23 Johanna: Un der Dande Berta hats schon gut 2425 Martha: gfalln;

26 Anna:

$\mathrm{OH}$ : ob und wie!

$$
\text { Der! Oh Gott }=e=\text { Gott }
$$

Beispiel 5:

(1)

\section{AK : EM 21/8}

\begin{tabular}{|c|c|}
\hline \multicolumn{2}{|c|}{ AK: EM 20/84 : 1 „Ganz umgänglich“ } \\
\hline 01 Marlies: & $\mathrm{Na}$ ich fand den Hübner-Voss ja \\
\hline 02 & sehr sympathisch also ich war ir- \\
\hline 03 & gendwie verblüfft daß ich jemand \\
\hline 04 & auf Anhieb (-) wie ich da rein- \\
\hline 05 & kommn bin hab irgendwie- \\
\hline 06 & $(3.0)$ \\
\hline $\begin{array}{l}07 \text { Sonja: } \\
08\end{array}$ & $\begin{array}{l}\text { Ha doch der isch ganz umgänglich; } \\
\text { (5.5) }\end{array}$ \\
\hline \multicolumn{2}{|l|}{ Beispiel 3: } \\
\hline \multicolumn{2}{|c|}{ AK : EM 21/84 : 1 „Karriere“ } \\
\hline $\begin{array}{l}11 \text { Karola: } \\
12\end{array}$ & $\begin{array}{l}\text { Ja un die die Schleifinger die macht } \\
\text { ja jetz Karriere in Osnabrück- }\end{array}$ \\
\hline $\begin{array}{l}13 \text { Sabine: } \\
14\end{array}$ & $\begin{array}{l}\text { Wie bitte! Die! Wie kommt se denn } \\
\text { doadezu, }\end{array}$ \\
\hline 15 Karola: & Ha so wie se s hier au gmacht hot \\
\hline
\end{tabular}


reitet und eingeleitet. Der Charakter der gewählten Evaluationen und Deskriptoren ist signifikanterweise in hohem Maß unspezifisch und indirekt. ${ }^{6}$

Indirektheit und Unspezifität der Bewertungen und Beurteilungen in der Präsequenz verweisen darauf, daß hier, diskret, eine Aktivität vorbereitet wird, die einen ambivalenten, ja, sozial betrachtet unter Umständen sogar einen ,gefährlichen' Nimbus hat. Am Gesprächsausschnitt „Sizilianisch“" (Beispiel 4) läßt sich exemplarisch veranschaulichen, wie in einer ganz, harmlos* klingenden ÄuBerung eine vorhandene Bereitschaft zum Klatschen zum Ausdruck gebracht, gleichzeitig aber eine Offenlegung dieser Absicht vermieden wird, somit eine Zurücknahme dieser Klatsch-Nachfrage ohne Gesichtsverlust im Rahmen der gegebenen interaktiven Möglichkeiten bestehen bleibt. Die assertorische Komponente der Äußerung ,Un der Dande Berta hats schon gut gfalln“ ist unüberhörbar. Hier wird nicht lediglich eine Information nachgefragt (im Sinne von: Wie hats der Tante Berta in Sizilien gefallen?'). Hier ist jemand bereits im Besitz gewisser Informationen (und sei es nur der Art, daß Tante Berta immer ,für Überraschungen gut ist ${ }^{\prime}$ ), stellt sich aber in gewisser Weise ,dümmer' als er ist, um mehr zu erfahren, als er bereits weiß. Das „Und“ zu Beginn der Äußerung verweist auf ein vorgängiges Wissen, mit dem „schon“ wird eine Annahme zum Ausdruck gebracht und eine Behauptung aufgestellt. Bezieht man prosodisch-paralinguistische Merkmale wie Intonation (leicht amüsierter Tonfall) und mimisch-gestisches Ausdrucksverhalten (leichtes Lächeln, spezifischer Blickkontakt) in die Analyse mit ein, so wird vollends deutlich, daß hier nicht die schon angenommene oder sogar gewußte Information (,daß es der Tante Berta gut gefallen hat') im Zentrum der Interaktion steht. Die Sprecherin macht mit ihrer Nachfrage den anderen auch ein Angebot, im Sinne von: ,Wenn ihr mehr wißt als ich, sagt es mir, es interessiert mich'. Die dadurch etablierte Insider-Outsider-Relation dient darüber hinaus dem Zweck, die besser Bescheidwissende/n zur Weitergabe ihrer Informationen, zur Demonstration ihres Wissens zu bewegen. Berücksichtigt man weiterhin, daß es sich in diesem Fall um die Mitglieder einer Familie handelt, in der ein Zurückalten von Informationen negativ interpretiert werden würde, so wird der ,Fishing-Charak-

\footnotetext{
${ }^{6} \mathrm{Vgl}$. „netter Mensch“ in ..Nichts Tolles“, Beispiel 1; vgl. auch „sympathisch“ im Text „Ganz umgänglich“, Beispiel 2.
}

ter' der sequenzinitiierenden Äußerung unüberhörbar. Auch die Wahl unspezifischer Deskriptoren wie "schon gut gfalln“ lädt den Interaktionspartner zu einer Präzisierung ein. Dennoch bleiben diesem andere Möglichkeiten offen, er muß nicht mit einer Klatschgeschichte reagieren, er kann die ihm bekannten Informationen, wenn schon nicht verweigern, doch auf eine, wertneutrale" Art und Weise weitergeben. Das Gespräch befindet sich gleichsam noch in der Schwebe. Der Klatschinitiator vermeidet durch die Indirektheit der Einleitung ein Offenlegen seiner Absichten und vermindert dadurch die Gefahr, als ,klatschhaft" oder ,klatschsüchtig' verurteilt zu werden. Dem Rezipienten einer solchen Äußerung wird damit die Möglichkeit zur ,diskreten“ Verweigerung eingeräumt. Ein Beispiel dafür gibt der Gesprächsausschnitt „Promotion“ (s. Bsp. 5). Hier enthält die erste Äußerung die erwähnten Modalisierungen und Animierungen, die von einem Interaktionspartner aber zurückgewiesen werden, indem dieser klarstellt, $\mathrm{da}$ B er selbst maßgeblich am klatschträchtigen Geschehen beteiligt war und ist (vgl. Z 09f.).

Die Unspezifität der Deskriptoren erweist sich auch in einem weiteren Punkt als funktional für die Aktivität des Klatschens: Durch die Wahl unspezifischer, ambiger und in aller Regel schwacher Bewertungen von Personen oder von deren Handlungen wird dem Interaktionspartner die Möglichkeit des Kundtuns einer abweichenden Einschätzung oder Beurteilung gegeben. Jemand, der ein „ganz netter Mensch“, „sympathisch“ oder „ganz umgänglich" ist, kann ja durchaus Eigenschaften aufweisen, die im Kontrast dazu stehen, und so ein Verhalten zeigen, das klatschwürdig ist, ohne da 3 dies seine Charakterisierung als ,ganz nett" umstoßen müßte. Indem derartige Charakterisierungen sehr vage und allgemein gehalten sind, bieten sie gerade die Möglichkeit zur Korrektur und sind damit für eine Evokation von Klatsch durchaus funktional.

Betrachten wir nun die reaktiven Äußerungen, die den Fortgang des Gesprächs entscheidend mitbestimmen, genauer. Für sie ist zunächst der bereits erwähnte Rekurs in pronominalisierter Form auf die Person des Klatschobjekts typisch, mit dem die Kenntnis der abwesenden Person bestätigt wird. Darüber hinaus zeigen alle Textausschnitte aber, daß auch in den zweiten Äußerungen Evaluationen enthalten sind. Die Beurteilungen der ersten und der zweiten Äußerung beziehen sich dabei jeweils auf denselben Referenten (Person, Situation, Ereignis, Aktivität). Auch dies macht wiederum 
deutlich, daß beide Interaktionspartner über eigenes direktes Wissen aus erster Hand über die besprochene Partei verfügen. Die Bezugnahme auf den Referenten in der zweiten Äußerung ist stets mit einer Bezugnahme auf die evaluative Komponente der ersten Äußerung verknüpft. ${ }^{7}$ Im Falle übereinstimmender Beurteilungen in der sequenzinitiierenden und der reaktiven Äußerung, wie sie z. B. in den Textsegmenten "Sizilianisch" (s. Beispiel 4) und „Karriere“ (s. Beispiel 3) vorwiegend durch Intonation und paralinguistische Phänomene zum Ausdruck kommen, ist die Klatsch-Bereitschaft häufig schon mit diesem Äußerungspaar hinreichend für beide Seiten dokumentiert. Damit kann die Klatschpräsequenz als ,erfolgreich' abgeschlossen gelten. Das aus dem Alltag vertraute Phänomen, daß manchmal ein ,leises Antippen' in Richtung Klatsch schon zum Erfolg führen kann, verdankt sich aber nicht der Konkordanz der Beurteilungen des Klatschobjekts allein. Konstitutiv für diesen - relativ kurzen, häufig nur aus einem ÄuBerungspaar bestehenden Präsequenztypus - ist eine personale Konstellation, die durch einen hohen Intimitätsgrad der Beteiligten und durch ein geringes Wissensgefälle gekennzeichnet ist, wie zum Beispiel bei Familienmitgliedern oder sehr guten Freunden. Hier ist zum einen auf Grund der bestehenden Vertrautheit die Gefahr einer negativen Sanktionierung der Klatschhaftigkeit relativ gering; zum anderen findet sich gerade in solchen Beziehungen sehr häufig ein Grundkonsens im Hinblick auf soziale und moralische Wertvorstellungen und Normen. Aber auch nicht-übereinstimmende Beurteilungen von Personen, oder von deren Handlungen, können als Auslöser für ein Klatschgespräch wirksam werden. Im Transkript „nichts Tolles“ (Beispiel 1) kommt es im Anschluß an einen Meinungsdissens zu einer Klatschgeschichte, die hier als Beleg für eine abweichende Meinung erzählt wird (vgl. ebenso Beispiel 2). In diesem Fall erstreckt sich die Klatschpräsequenz über mehrere Redezüge, ${ }^{8}$ denn Nicht-Zustimmungen werden in aller Regel durch abgeschwächte Zustimmungen, Pausen und/oder Nachfragen eingeleitet. ${ }^{9}$ Damit verschiebt sich zwangsläufig der Beginn der nachfolgenden Klatschgeschichte. Bei Meinungsverschiedenheiten sind abweichende ne-

\footnotetext{
'Vgl. zum Beispiel Gesprächssegment "Nichts Tolles“: Monika sagt:.... netter Mensch ...", Erich sagt: „ganz nett . . .“; vgl. auch Transkript „Ganz umgänglich“: Marlies sagt:., . . . sehr sympathisch . . “, Sonja sagt: ,.. . ganz umgänglich .
}

gative Beurteilungen meist sequentiell weiterführend. Die Frage nach der Existenz, positiven Klatschs' können wir aufgrund des uns vorliegenden Materials nicht positiv beantworten: In allen Fällen sind es mehr oder weniger negativ bewertete Handlungsweisen, die zum Klatschen stimulieren. ${ }^{10}$

Halten wir fest: Die Klatschpräsequenz besteht in jedem Fall aus einer klatschinitiierenden und einer reaktiven Äußerung. Mit diesem Äußerungspaar wird eine abwesende Person (das zukünftige Klatschobjekt) als den Anwesenden bekannt identifiziert. Die hohe Indexikalität der sequenzinitiierenden wie der reaktiven Äußerungen verweist dabei auf die enge soziale Beziehung der Beteiligten. In der Klatschpräsequenz werden Meinungen über Abwesende ausgetauscht und Bewertungen vorgenommen. Die Wahl unspezifischer evaluativer Deskriptoren, die spezielle Intonation und mimisch-gestisches Verhalten führen zu einer indirekten und diskreten Initiierung. Zunächst muß die Bereitschaft aller zum Klatsch intersubjektiv ratifiziert werden. Im Verlauf und durch die Präsequenz findet ein koordinierter Einstieg ins Gespräch statt, die Rollen von aktiv und passiv Klatschenden werden interaktiv ausgehandelt, und damit wird die für den Klatsch geeignete Situation etabliert. Vertrautheit und wechselseitige Kenntnis sind dabei notwendige Bedingungen, ohne die kein Klatsch zustande kommt, sie allein reichen aber nicht aus. Jede aktuelle Interaktionssituation muß jeweils neu als geeignet für den Austausch von Klatsch definiert werden; dies kann unter bestimmten Umständen sehr rasch und, ökonomisch

${ }^{8}$ Im Gesprächssegment „Ganz umgänglich“ stimmt die zweite Sprecherin, Sonja, nach einer Pause von drei Sekunden der ersten Sprecherin, Marlies in abgeschwächter Form zunächst zu. Die Drastik der Abschwächung deutet schon einen Dissens an; nachdem in einer darauffolgenden fünfsekündigen Pause der Redezug vakant bleibt, fährt Sonja mit einer Explikation ihrer implizit bereits angedeuteten negativen Einschätzung des Klatschobjekts fort. Ähnlich im Transkript „Nichts Tolles“; auch hier wird der Rezipient einer Beurteilung in der Folge durch den ersten Sprecher zum Erzählen einer Klatschgeschichte animiert.

${ }^{9}$ Vgl. dazu ausführlich Anita Pomerantz (1975), die sich mit dem Problem von ,Nicht-Zustimmungen' im einzelnen beschäftigt.

${ }^{10}$ Für Geschichten, die zur Mehrung des Ruhmes von jemanden erzählt werden, gilt in aller Regel keine Geheimhaltungspflicht 
erledigt werden, es kann aber auch in ,elaborierter' Form im Rahmen eines Meinungsaustauschs erfolgen. " Die Einleitungssequenz bereitet den Klatsch vor, führt in spezifischer Weise zu ihm hin und ist retrospektiv betrachtet ein Teil von ihm. Die Form der indirekten Einleitung, die dabei gewählt wird, ist ein Ausdruck der Ambiguität, die den Klatsch als kommunikatives Genre der Faceto-Face-Kommunikation kennzeichnet. Die Spannung zwischen öffentlicher Ächtung und weitverbreiteter Praxis findet ihren Niederschlag auch in der konversationellen Praxis, die von seinem ,Sitz im Leben' (um mit A. Jolles zu sprechen) geformt wird. Andererseits vermag der Klatsch diese seine Stellung durch die seinen Verlauf prägende Aktivität der indirekten Einleitung selbst mit zu beeinflussen. Die Vorsicht, mit der das Geschäft des Klatschens betrieben wird - und betrieben werden muß - ist nicht nur ein konstitutiver Bestandteil, sie erhöht auch seine Attraktivität und trägt ganz wesentlich zu dem Vergnügen bei, das der Klatsch auch und vor allem den Klatschenden bereitet. Die Ächtung der Moralisten (im Alltag wie in der Philosophie), die sich im wesentlichen darauf gründet, daß im Klatsch vertrauliche Informationen weitergegeben, Geheimnisse verraten und Privates öffentlich gemacht wird, hat zwar ihren guten Grund, sie trifft aber nur zum Teil zu. Denn wie sich auch an der Präsequenz ablesen läßt, findet die Informationsdistribution nur in beschränktem Rahmen, im personell strikt reglementierten Kreis statt. Informationen werden nur an solche Personen weitergegeben - und sind überdies auch nur für diese ,interessant ${ }^{\text {' }}$, , die bereits im Besitz gewisser Vorkenntnisse sind und zum jeweiligen Klatschobjekt in einer vertrauten Beziehung stehen. Gleichwohl wird die Tatsache, daß es sich um die Weitergabe, vertraulicher" Informationen handelt, im Klatsch mitreflektiert und findet sich in der Pragmatik des Genres wieder.

Betrachten wir nun die spezifischen Merkmale dieser vertraulichen Mitteilungen und die interaktive Prozedur ihrer Übermittlung im einzelnen.

\section{Die Klatschgeschichte}

Im Verlauf der stark dialogisch strukturierten Klatschpräsequenz findet, wie wir gesehen haben, ein koordinierter Einstieg in das Gespräch statt,

11 Gail Jefferson (1978 : 224) schreibt: „Entry into a story from turn-by-turn talk can be done economically or elaborately."

die Rollen von Sprecher und Zuhörer beziehungsweise Klatschproduzent und Klatschrezipient werden ausgehandelt und wechselseitig ratifiziert, gleichzeitig wird damit auch die jeweilige Interaktionssituation als geeignet für den Austausch von Klatsch definiert. Die Klatschgeschichte im engeren Sinn beginnt in dem Moment, in dem der durch interaktive Prozeduren zu einem solchen gewordene Klatschproduzent mit seiner Geschichte beginnt. Diese wird also, wie wir daran sehen, nicht als ein ,plötzlicher Einfall' ins Gespräch eingebracht, sondern sie steht im Zusammenhang mit den aufeinander abgestimmten Redezügen der Präsequenz. Im Textausschnitt „Umgänglich“ wird im Anschluß an die Präsequenz das Verhalten des Klatschobjekts an Hand mehrerer Beispiele illustriert: Sonja, die Klatschproduzentin, beschreibt Situationen, in deren Mittelpunkt das wenig ,umgängliche' Verhalten ihres Chefs steht. Die Redebeiträge der Klatschrezipientin Marlies beschränken sich in dieser Phase des Gesprächs auf aufmerksamkeitsmarkierende „Mhm's“, kurze Nachfragen zum richtigen Verständnis oder zustimmendes Lachen.

\section{Beispiel 6:}

AK : EM 20/84 : 10-12 „Ganz umgänglich“

09 Sonja:

10

11

12

13

14

20

21

22

23

24

25

35

36

37 Marlies: 3839 Sonja:

40

41

47

48
Mein s kommt er abr scho au so manchmal s Gfühl ghabt also : : ich wär zu seim Privatvergnügn do abgschtellt also: (-) wenn er halt pfeift daß ich dann (-) gleich Stramm-Stehen sollte

Do hat mer heut um zwölfe n Termin da isch er ebbä widr so um elfe rum kam er irgendwann zu mir rein do war ich grad am telefoniere do: lief er zweimol raus (---) kam widr rein so: $(-)$

irgendwie hat er halt so $\mathrm{n}$ Loch ghabt was weiß ich,

No sollsch du da sein $\left[\begin{array}{l}\text { dich mit ihm unter } \\ \text { ja }\end{array}\left[\begin{array}{l}\text { haltn. } \\ \text { ja }\end{array}\right.\right.$

Und nochher war er ja heut mit Essn holn dran

hab i gsagt „i kann au net an alles denkn" 
49

50 Marlies:

Hnhhnhehhnhi $\begin{aligned} & \text { hnehennhehh. . . } \\ & \text { hehhehehhhehh. . }\end{aligned}$

((gemeinsames Lachen))

51

52 Marlies:

53

54 Sonja:

Geht ja nett zu bei euch wie beim altn Ehepaar jahehehhenhnhahaha

Das Ende der Klatschgeschichte wird von der Klatschproduzentin markiert und von der Rezipientin ratifiziert. Ein abschließendes Lachen, in das die Zuhörerin einstimmt, gefolgt von einer Pause und einer resümierenden Bemerkung, macht den Abschluß des stark monologisch geprägten Abschnitts der Klatschkommunikation, in dem die Klatschproduzentin Sonja diverse Episoden aus dem Berufsalltag mit ihrem neuen Chef geschildert hat, deutlich.

Lachen, Pausen, Floskeln wie „jaja“, „,nun gut" oder "soso" etc. (jeweils mit absinkender Intonationskontur) werden, wenn sie einen Redezug für sich allein bilden, vom Sprecher häufig als Mittel benutzt, um anzuzeigen, daß er nichts mehr oder nichts Neues mehr zu sagen hat. ${ }^{12}$ Ganz ähnlich sieht das Ende der Klatschgeschichte in anderen Gesprächen aus; ein weiteres Beispiel:

\section{Beispiel 7:}

\section{AK : PM 14B/84 : 6 „Sizilianisch“}

52 Martha: Secht se "No ben e nachts raus no 53 hanne immer nemme gwißt wars ${ }^{\circ h}$ 54 bei welcher Dier daß ${ }^{\circ}$ ens Klo nei$55 \quad$ geht $^{\circ}$ no ben e halt mol beim Salva56 dore drin glandet,,Hihihehhhn57

58 Johanna:

59 Lea:

60 Anna:

61

62

63

64 hhnhihh [ ihehhnehhh in dr Nacht ((gemeinsames Lachen))

Mit solchen Abschlußmarkierungen (Floskeln, Resümmees, Lebensweisheiten, Redewendungen), die Schegloff/Sacks (1973: 306) „Pre-closings“ nennen, übernimmt der Sprecher einen Redezug, dessen Aufgabe es ist, den Turn an den Interaktionspartner weiterzugeben, diesem anzuzeigen,

${ }^{12}$ Vgl. E. Schegloff/H. Sacks (1973 : 304-306) daß er selbst den nächsten Redezug nicht mehr für sich beansprucht, da er erst einmal nichts mehr weiter zu sagen hat. Das Gesprächsthema wird wieder in die allgemeine Unterhaltung eingegliedert, in dem Sinne, daß die stark monologisch strukturierte Klatschgeschichte zur ,allgemeinen' Kommentierung freigegeben wird. Dementsprechend schließt sich an die jeweilige Klatschgeschichte auch eine Kommentierungs- und Generalisierungsphase an, an der sich Klatschproduzent und Klatschrezipient aktiv beteiligen.

Doch zunächst zurück zur Klatschgeschichte, zu ihren inhaltlichen und stilistischen Merkmalen:

Klatschmitteilungen, so die Minimaldefinition, betreffen die persönlichen Angelegenheiten eines guten Bekannten, Freundes oder Familienmitglieds und Verwandten. Um beim Zuhörer auf Interesse $\mathrm{zu}$ stoßen, müssen sie in irgendeiner Form ein Element von Neuheit enthalten. Die „Neuheit der interessanten Materie" (Goethe ${ }^{8} 1973$ : 141). stimuliert und provoziert die Erzählung. Jemand ist im Besitz einer Information, an deren Verbreitung er - aus welchen Gründen lassen wir zunächst dahingestellt - interessiert oder zu der er zumindest bereit ist. Handelt es sich um eine vertrauliche Information, so stellt der Erzählende zum einen seine exklusive Beziehung zur abwesenden Person, die die Klatschmitteilung betrifft, unter Beweis; er demonstriert zum anderen aber auch sein Vertrauen zum aktuellen Gesprächspartner, da er diesen an seinem Wissen teilhaben läßt.

Klatschgeschichten handeln meist davon, wie man sich benehmen, bzw. wie man sich nicht benehmen soll. Fragen der Schicklichkeit, des Anstands, der Moral und ,guten Sitten' sind ebenso ihr Thema wie soziale und vor allem sexuelle Regelverletzungen. Im eingangs vorgestellten Gesprächsausschnitt „Karriere“ sind es die erotischen Karrierehilfen einer Kollegin, die Mißfallen erregen und Klatsch stimulieren; im Gespräch „Ganz umgänglich" ist es das chauvinistische und ausbeuterische Verhalten eines Chefs, und im Gespräch „Sizilianisch" schließlich steht das unkonventionelle Verhalten eines Familienmitglieds im Zentrum der Unterhaltung.

Ob über Charaktereigenschaften, Fragen des Temperaments, physische Absonderlichkeiten oder soziale Regelverletzungen: Geklatscht wird zumeist und in erster Linie über Ereignisse und Verhaltensweisen, die als auffällig, erstaunlich, befremdlich, unkonventionell, beunruhigend oder falsch angesehen werden. Gegenstand eines Klatschgesprächs sind Ereignisse oder Verhaltensweisen, die 
vom Klatschproduzenten entweder selbst beobachtet oder miterlebt wurden oder über die er von einer beteiligten Person informiert wurde. Im allgemeinen wird ein Ereignis (eine Handlungsweise) beschrieben, bewertet und kommentiert. Mit den Mitteilungen findet nicht nur eine Transmission neuer Informationen statt, sondern es werden subjektive Interpretationen von Ereignissen übermittelt, die vom jeweiligen Sprecher auch als solche gekennzeichnet werden. Der Klatsch lebt auch von einer häufig vorgenommenen impliziten moralischen Kontrastierung zwischen dem Erzähler auf der einen, und dem Protagonisten der Erzählung auf der anderen Seite. ${ }^{13}$ Die wertende Wiederbelebung eines Ereignisses, die im Klatsch stattfindet, bringt immer auch die je eigene Sichtweise des Klatschproduzenten zum Ausdruck. Die Art und Weise, wie ein Ereignis präsentiert wird, hängt aber auch von der (gewußten oder vermuteten) Haltung des Rezipienten ab. Vorsichtig und schwach fallen die Evaluationen dann aus, wenn sich der Sprecher selbst unsicher ist, wie er ein bestimmtes Verhalten beurteilen soll, oder aber auch dann, wenn für ihn die Beurteilung durch den Rezipienten nur vage abschätzbar ist. Dies stellt allerdings nur bedingt ein Problem für den Handelnden dar, da Evaluationen in der Klatschgeschichte generell nur selten vom Sprecher explizit vorgenommen werden. Die Wirkung ,kunstvollen Erzählens und Klatschens beruht ja nicht zuletzt darauf, daß dem Zuhörer die Extraktion der (gewünschten oder nahegelegten) Schlußfolgerungen überlassen bleibt. Ein bedeutungsvolles Schweigen an der entsprechenden Stelle, eine dezente Anspielung, eine halbe Vermutung oder ein schwebendes Offenlassen sind Kunstgriffe, durch die implizite Wertungen im Klatsch wirksam werden. Jede Form zu großer Deutlichkeit würde deren Wirkung ebenso schmälern wie Übertreibungen die Glaubwürdigkeit des Klatschproduzenten beeinträchtigen könnten. In der Fähigkeit, aus nichts etwas zu machen', zeigt sich nicht zuletzt die kreative Leistung und der Einfallsreichtum der Klatschenden. Bend festhalten, wird durch eine spezifische Art und Weise der Verknüpfung neuer und evaluativer
Mit der Klatschgeschichte, so läßt sich abschlie-

Elemente ein Verhalten rekonstruiert und bewertet, auf dessen Basis ein moralischer Charakter des Klatschobjekts behauptet und etabliert wird. Von den in der Klatschgeschichte präsentierten singulären Handlungsweisen werden verallgemeinernde Schlüsse auf die Person und den Charaktertypus des Klatschobjekts gezogen; ${ }^{14}$ diese werden in der sich anschließenden Klatschnachsequenz im Blick auf soziale Typen generalisiert und im diskursiven Meinungsaustausch expliziert.

\section{Die Klatschnachsequenz}

Wie wir bei der Betrachtung der Klatschgeschichte gesehen haben, schließt der aktiv Klatschende seine jeweilige Geschichte mit einem klaren Hinweis auf deren Ende ab, was vom passiv Klatschenden verstanden und ratifiziert wird. Die sich daran anschließende Klatschnachsequenz wird - ähnlich wie die Klatschpräsequenz - von einem wieder stärker dialogisch strukturierten Meinungsaustausch bestimmt. Beide, Klatschproduzent und Klatschrezipient, nehmen wieder aktiv am Gespräch teil. Die Beurteilungen des Klatschobjekts, wie sie in der Präsequenz angelegt und in der Geschichte implizit enthalten sind, werden in der Nachsequenz elaboriert, sie werden hier zum Thema der Konversation gemacht. Betrachten wir zunächst den Fortgang der Gesprächssegmente „Ganz umgänglich“ und „Sizilianisch“:

\section{Beispiel 8:}

\begin{tabular}{|c|c|}
\hline AK : EM & \\
\hline 58 Sonja: & $\begin{array}{r}\mathrm{Ja} \text { also die }=\mathrm{die}=\mathrm{die}= \\
(1.6)\end{array}$ \\
\hline 60 & die Schowis $=$ \\
\hline 61 Marlies: & $=\mathrm{S}$ is unglaublich weisch \\
\hline $\begin{array}{l}62 \text { Sonja: } \\
63 \\
64\end{array}$ & $\begin{array}{l}\text { Un die }(-) \text { also: sobald de dene } \\
\text { irgendwo } n \text { klein Finger gibsch dann } \\
\text { packn se zu }\end{array}$ \\
\hline 68 Marlies: & $\begin{array}{l}\text { so ohne }(-) \text { würd doch würdn } \\
\text { [ würdn doch mir nie machn }\end{array}$ \\
\hline $\begin{array}{l}70 \text { Sonja: } \\
71 \text { Marlie } \\
72\end{array}$ & $\begin{array}{l}\text { mhm mhm } \\
\text { auch nur im umgekehrten Fall } \\
(2.0)\end{array}$ \\
\hline
\end{tabular}

${ }_{13}^{13}$ Im Gesprächssegment "Sizilianisch" verweist Anna wiederholt darauf, daB Tante Berta die kolportierte ,peinliche` Situation hätte vermeiden können, wenn sie ihren, Annas, Ratschlägen gefolgt wäre und sich demgemäß richtig verhalten hätte. glickliche Nadur Dande Berta" und in Beispiel 6: „Geht ja net zu bei euch wie bei einem alten Ehepaar $^{*}$.
${ }^{14} \mathrm{Vgl}$. Transkript „Sizilianisch“, Beispiel 7: „Hot scho e 


\section{Beispiel 9:}

AK : PM 14B/84 : 6 ,Sizilianisch“

66 Martha: Au beim Flug also (--) scheints pu-

67

68 Anna:

69 Lea:

70

71

76 Martha: Aus lauter Freude am Erläbn. . .

Tante Berta, wird hier deutlich, gehört zu den Leutn, die aus Freude am Erleben Dinge tun können, die ihr hohes Alter sonst nicht erlauben würde; der Hübner-Voss ist ein Chef, der von einem erwartet, daß man sich stets nach ihm richtet, der Frauen ausnutzt und daher zur Gruppe der männlichen Chauvinisten gehört. Das jeweils individuelle und singuläre Verhalten wird hier in der kommentierenden Rede zu einem für die jeweilige Person typischen erklärt. Typisch auch insofern, als es für eine spezifische Personengruppe als typisch angenommen wird (für alte Frauen oder für chauvinistische Männer).

Es findet eine soziale Generalisierung statt, die auf die Etablierung eines individuellen Charakters in der Klatschgeschichte, die Etablierung eines bestimmten Charaktertyps folgen läßt. Die Beurteilung und Bewertung eines aktuellen Verhaltens wird in Beziehung gesetzt zu allgemeinen Handlungsweisen einer Gruppe von Personen, als deren Mitglied die betreffende Person betrachtet wird. In diesem Prozeß erhalten und verändern soziale Typen ihr Gesicht. Die Evaluationen und Kommentare der Nachsequenz haben zumeist einen moralischen Anspruch. Sie beschreiben das Verhalten von Personen im Hinblick auf ,das Wohl aller', auf soziale und moralische Normen, die eine intersubjektive Praxis regeln und die als gut und wünschenswert vor dem Hintergrund einer - im jeweiligen Lebenskontext - für sinnvoll erachteten Lebenspraxis angesehen werden. ( Vgl. dazu den nachfolgenden Exkurs.) Die Klatschnachsequenz hat innerhalb einer Unterhaltung immer auch eine themenbegrenzende Funktion; im Anschluß an die Kommentierungsphase wird das Gespräch entweder mit einem neuen (Klatsch-)Thema fortgesetzt oder aber mit den entsprechenden konversationellen Praktiken (Verabschiedung) zum Abschluß gebracht.

\section{Exkurs: Soziale und moralische Regeln im Klatsch}

Beim Klatsch ist der Klatschende zunächst damit beschäftigt, zu beschreiben, was ein Protagonist getan hat; im Anschluß daran wird das beschriebene Verhalten kommentiert, Erklärungen werden angeboten, und es wird über die Gründe oder Motive des Protagonisten spekuliert, Verhalten wird interpretiert, verurteilt oder gerechtfertigt. Im Zusammenhang mit diesen Erklärungen findet ein Rekurs auf - praktische, soziale, moralische Regeln statt; diese sind ein Teil der Sprache, mit der das Verhalten anderer verständlich gemacht, gerechtfertigt, kritisiert oder verurteilt wird. Im Klatsch wird Regelwissen üblicherweise durch den Bezug auf vom Normalen abweichendes, auffälliges Verhalten aktiviert. Gerade die Eigenartigkeit eines Verhaltens stimuliert ja den Klatsch darüber, was eigenartig an ihm ist. Regeln werden herangezogen, um ein Verhalten beurteilen zu können; normalerweise fangen wir gerade dann an zu klatschen, wenn etwas passiert, was unseren Erwartungen nicht entspricht. Die Zuschreibung eines Motivs oder einer Erklärung dient dabei als Begründung für die Rechtfertigung, bzw. häufiger noch für die Verurteilung bestimmter Verhaltensweisen. Dabei wird der Versuch gemacht, Handlungsweisen verständlich zu machen in bezug auf bekannte, übliche oder erwartbare Verhaltensund Handlungsweisen. Man kann aber nur dort „aus Überlegung“ handeln, wo es geltende Standards des Angemessenen gibt, an denen man sich orientieren kann. Klatsch ist in dieser Sichtweise also eine Aktivität, in der ein aktuelles Verhalten verbal verständlich und damit einer Anwendung von Regeln zugänglich gemacht wird. Die spezifische Art und Weise, in der im Klatsch Beurteilungen anderer vorgenommen werden, die Indirektheit und Unspezifität der gewählten (moralischen) Evaluationen, unterscheidet das Klatschgespräch gerade auch von anderen Formen des Redens über Leute. Um im Klatsch als Hintergrund für eine Bewertung herangezogen zu werden, muß eine Regel aber weder in den Gedanken der Leute, die sich implizit auf sie beziehen, als solche präsent sein, noch muß sie von denen, die sie anwenden, ausdrücklich formuliert werden können. Regeln, die zu Erklärungen (oder Kommentaren) im Klatsch herangezogen werden, sind häufig $\mathrm{Be}$ standteile eines Gewohnheitswissens: „Die Elemente des Gewohnheitswissens werden nicht mehr als Wissenselemente, als selbständige Erfahrungsthemen erfaßt, sondern sind im Horizont des Erfahrungswissens mitgegeben." (s. Schütz/Luckmann 1979 : 173). Gewohnheitswissen ist zwar aus erworbenen und spezifischen Wissenselementen entstanden (im Unterschied $\mathrm{zu}$ den universellen Elementen des Wissensvorrats), auf Gewohnheits- 
wissen kann aber automatisch immer wieder als problemlos zuhandener Bestand zurückgegriffen werden. Im Klatsch werden nun in erster Linie nichtregelkonforme Verhaltens- oder Handlungsweisen einer Interpretation zugänglich gemacht. Die besondere Form der Interpretation, wie sie in Gestalt und Form der Klatschkommunikation zum Ausdruck kommt, erlaubt aber einen differenzierten Umgang mit Regelabweichungen. Die Regeln, die hier zur Diskussion stehen, sind - wie schon bemerkt - praktischer, sozialer und vor allem moralischer Natur. Moralische Regeln haben ihrer Natur nach einen universalistischen Anspruch. Um aber im Alltag daraus praktische Handlungskonsequenzen ableiten zu können, müssen sie auf den jeweiligen Fall angewendet werden; nur so kann man herausfinden, ob eine konkrete Handlung verboten, toleriert, ermutigt oder verlangt wird. Kulturelle Regeln, seien sie ethischer oder sozialer Natur, werden nicht nur in der Kindheit gelernt; mit der Befolgung bestimmter Regeln lernen wir auch, wie wir über Verhaltensweisen reden können, als Teil einer Einübung in eine interpretative Praxis, die es uns erlaubt, uns als, soziale Wesen “ zu verhalten. Die Anwendung und Erprobung moralischer und sozialer Regeln ist ein Teil unseres alltäglichen Handelns, und Klatsch ist eine Methode, mit deren Hilfe Menschen im Alltag ihre moralischen Wahrnehmungen nach außen verlagern, öffentlich machen, sie damit auch erproben und der Überprüfung durch andere zugänglich machen können. Die im Klatsch stattfindende implizite moralische Kontrastierung zwischen Protagonist und Erzähler läßt auch eine Darstellung der eigenen Positionen zu. Der Klatsch wird sowohl von der Identität des Erzählers als auch von der Identität des Zuhörers beeinflußt. Der dialogische Charakter der Klatschkommunikation insgesamt macht auch - in gewissen Grenzen - neue Regelinterpretationen und damit Regeländerungen möglich, indem die Bedingungen der Regelanwendung zum Beispiel neu definiert werden. Dieselben moralischen Regeln lassen ja nicht immer nur eine Handlungsweise zu, sondern werden oft zur Legitimation ganz verschiedener und oft sogar widersprüchlicher Verhaltensweisen herangezogen. Eine gemeinsame Sichtweise moralischer oder kultureller Regeln dient aber (im Hintergrund) als Ausgangspunkt der Betrachtungen; gäbe es diese ,gemeinsame Perspektive' nicht, wäre es nicht möglich, bestimmte Handlungsweisen als, gut" oder ,schlecht", als gutzuheißend oder ablehnenswert zu beurteilen. Klatschen ist eine Möglichkeit, auf der Basis des, gesunden Menschenverstands' morali- sches Wahrnehmungsvermögen nach außen zu verlagern, zu dramatisieren und ihm eine konkrete Form zu geben. Im Klatsch über andere werden eigene moralische Sichtweisen angeboten und typischerweise als vernünftig oder richtig akzeptiert. Auf der Basis einer Billigung durch die Mitmenschen wird die Faktizität moralischer Regeln hergestellt. Der Anschein der Übereinstimmung, des Meinungskonsenses, bestärkt den Alltagsmenschen darin, daß die Gründe seiner moralischen Urteile außerhalb seiner persönlichen, individuellen Person liegen, von anderen geteilt werden, und damit unabhängig von seinen je eigenen persönlichen Wünschen, Überzeugungen und Interessen sind. "This taken-for-granted-assumption of the externality - objectivity - of the moral order is not only a consequence of gossip but a presupposition. Gossip, then, both presupposes and constructs an objective moral order . . . Evaluative talk of which gossip is an important part, spells out the common grounds of our social life." (s. Sabini/Silver 1982 : 102).

\section{Die Realisierungsformen}

Mit der bisherigen Analyse der sequentiellen Folge von Klatschpräsequenz, Klatschgeschichte und Klatschnachsequenz konnten die autonomen, weitgehend kontextunabhängigen Konstituenten dieses kommunikativen Genres bestimmt werden. Zum Abschluß soll nun ein Blick auf die konkreten Realisierungsbedingungen zeigen, welche kontextsensitiven Merkmale diese Art der Face-to-FaceKommunikation aufweist. Für die alltägliche Praktizierung dieses kommunikativen Genres spielen die jeweiligen Umstände, unter denen es realisiert wird, eine große Rolle. Die Analyse der Abfolgeordnung von Klatschgesprächen hat gezeigt, daß bei gleichbleibenden autonomen Merkmalen der Einstieg in den Klatsch ,ökonomisch' oder ,elaboriert‘ vollzogen werden kann, die autonomen Merkmale der Klatschpräsequenz ändern sich dadurch nicht. Was sich aber ändert, sind die Realisierungsbedingungen, und mit diesen ändert sich auch, wie wir sehen werden, der Charakter, den die Aktivität Klatsch annimmt.

Relativ rasch und ökonomisch erfolgt ein Einstieg in die Klatschgeschichte dann, wenn der Intimitätsgrad und die Vertrautheit der beteiligten Personen untereinander sehr hoch und das geteilte Wissen umfassend und gegenwärtig ist. Kennzeichnend für diese Form der Einleitung ist ein Meinungskonsens im Hinblick auf die Beurteilungen (der Handlungs- 
weisen) des Klatschobjekts. Eine elaborierte Gestalt nimmt die Präsequenz in den Fällen an, in denen der Gesprächspartner eine vom ersten Sprecher abweichende Meinung über die abwesende Person hat. Im Falle eines Meinungsdissens erstreckt sich die Eröffnungssequenz meist über mehrere Redezüge und endet in aller Regel mit einer (expliziten oder impliziten) Aufforderung an die ,nicht-zustimmende' Partei zu einer Begründung ihrer Beurteilung. Klatschgeschichten werden in diesen Begründungszusammenhang eingebettet, sie werden als Beleg für etwas erzählt und erhalten damit im weitesten Sinn den Status eines Arguments; allerdings den Status eines besonderen Arguments: Geklatscht wird, ob diskordant oder konkordant, immer auch zum und mit Vergnügen. Die Lust am Klatsch, das Vergnügen, das er bereitet, bestimmt ganz wesentlich seinen Charakter. Während dies im Falle des, diskordanten' Klatsches von diskursiven Strukturen mehr oder weniger überlagert wird, tritt es beim ,konkordanten' oder ,geselligen' Klatsch in aller Deutlichkeit zu Tage. Klatschgeschichten werden vorwiegend zur Unterhaltung, zum gemeinsamen Amüsement erzählt. Im vertrauten Kreis von Freunden, Bekannten oder Verwandten wird über Dinge gesprochen, ,die einen eigentlich nichts angehen', die die Privatsphäre Dritter betreffen, die vertraulich und damit gerade so interessant sind. ${ }^{15}$,Gerede nennt dies Heidegger, und bei Sören Kierkegaard (1959: 308) ist es das ,Geschwätz', „das dazu führt, das Menschsein immer unbedeutender zu machen." Dagegen läßt sich mit Georg Simmel ( $\left.{ }^{4} 1984: 63\right)$ einwenden, „daß auch das Erzählen von Geschichten, Witzen und Anekdoten, so oft es auch ein Lückenbüßer und Armutszeugnis sein mag, doch auch einen feinen Takt zeigen kann, in dem alle Motive der Geselligkeit anklingen." Allerdings unterscheidet sich der Klatsch (auch der gesellige) in einem Punkt entscheidend von der

\footnotetext{
Auf den journalistischen Klatsch in Zeitungen oder Illustrierten, der nur durch eine Mißachtung des für den mündlichen Klatsch gültigen Gebots der personalen Restriktion möglich ist, trifft die Heideggersche Kennzeichnung des ,Geredes' eher zu. Journalistischer Klatsch, der etwas öffentlich macht, von dem er behauptet, es sei privat, gibt bekannt, daß er im Besitz von Wissen sei und behauptet eine schier universelle Verständlichkeit des Lebens anderer Leute. Liest man ihn, so erlangt man die vergängliche Überzeugung, Insider-Wissen zu besitzen - ein Vergnügen, das allerdings mit Klatsch in all seinen Variationen verknüpft ist.
}

rein „geselligen Unterhaltung“, wie Simmel sie im Auge hat, und die er als eine beschreibt, „die jenseits aller individuellen Intimität, jenseits des rein Personalen steht, das sich nicht in die Kategorien der Geselligkeit fügen will. Aber dennoch ist dieses Objektive nicht um seines Inhaltes, sondern um des Geselligkeitsinteresses vorgebracht; daß dieses gesagt und aufgenommen wird, ist kein Selbstzweck, sondern ein bloßes Mittel für die Lebendigkeit, das Sichverstehen, das Gemeinschaftsbewußtsein des Kreises." (s. Simmel 1984 : 63). Der Klatsch steht aber gerade nicht „jenseits jenes rein Personalen“, im Gegenteil, das Individuelle und Besondere ist sein Thema, und deshalb klatscht man nicht mit fremden Personen über die persönlichen Angelegenheiten eines Freundes oder Familienmitgliedes. Man klatscht aber im vertrauten Kreis der Familie oder mit guten Freunden, und hier dient der Klatsch zum einen der Unterhaltung, zum anderen aber auch der Schaffung des von Simmel so betonten „Gemeinschaftsbewußtseins des Kreises" und der Etablierung eines „Wir-Gefühls“. Anders als es häufig unterstellt wird, geht es im Klatsch nicht nur darum, um des Redens willen zu reden, es geht auch nicht nur darum, Insider-Wissen weiterzugeben und Geheimnisse öffentlich zu machen; es geht auch und vor allem darum, Verhalten zu interpretieren, Erklärungen für nicht übliche, nicht regelkonforme Handlungen anzubieten und diese damit auch einer gemeinsamen Beurteilung zugänglich zu machen. Dies wird mehr noch als beim rein ,geselligen' beim ,diskursiven' Klatsch deutlich, da hier ein Konsens der Evaluationen erst im Laufe des Gesprächs erreicht und damit interaktiv produziert wird. In allen uns vorliegenden Textbeispielen sind sich Klatschproduzent und Klatschrezipient in der Kommentierungsphase der Klatschnachsequenz im Hinblick auf die Bewertung bestimmter Handlungsweisen einig. Dies unabhängig davon, ob in der Klatschpräsequenz unterschiedliche oder übereinstimmende Evaluationen vorgenommen wurden. Dies ist nur vor dem Hintergrund dessen möglich, daß diese Evaluationen in hohem $\mathrm{Maß}$ indirekt und unspezifisch in der Präsequenz gehalten sind. Erst in der Nachsequenz, im Prozeß sozialer Generalisierungen, werden Werturteile explizit gemacht und damit ihrer Vagheit beraubt. Die individuelle Person wird aber auch im Falle explizit negativer Beurteilungen nur selten insgesamt verurteilt. Auch dann, wenn, wie wir dies beim Textausschnitt „Ganz Umgänglich“ (s. Beispiel 4, 6 und 8) sehen können, das Verhalten einer Person in bestimmten Situationen ein- 
deutig negativ beurteilt und diese im Zusammenhang damit als Mitglied einer wenig sympathischen Personengruppe präsentiert wird, kommt dennoch auch hier ein Moment der Sympathie zum Tragen, das auf die Realisierung von Klatsch einen ganz entscheidenden Einfluß hat und in direktem $\mathrm{Zu}$ sammenhang mit dem spezifisch unspezifischen Charakter der Evaluationen steht.

Beispiel 10:
AK : EM 20/84 : 12-13 „Ganz umgänglich“
73 Sonja:
74
75
76
77
Weiß es au nette ( - ) Mein anderer- seits wenn ich morgends komm no hat er mir an Tee gmacht (-)
Do macht er sein Kaffee und no stellt er $\mathrm{n}$ Tee auf derweil
87 Sonja 88

Die positive Wendung, die dieses Gespräch noch nimmt, ist nicht zuletzt vor dem Hintergrund dessen zu sehen, daß Sonja weiterhin mit ihrem Chef, dem Hübner-Voss, zusammenarbeiten muß und eine völlige Ablehnung seiner Person im Hinblick auf das Alltags- und Berufshandeln zu schwierigen Konsequenzen führen könnte. Das in der spezifischen personalen Konstellation, in der es überhaupt nur zum Klatsch kommt, sich widerspiegelnde soziale Beziehungsgeflecht der beteiligten Personen findet seinen pragmatischen Ausdruck in der den Klatsch mitkennzeichnenden Sympathiekomponente, die für die Realisierung von Klatschgesprächen nicht ohne Bedeutung ist. Beim Klatsch wird ja - in aller Regel - über Bekannte, Freunde oder Familienmitglieder gesprochen, Informationen über deren persönliche Angelegenheiten werden aber nur an Freunde (Bekannte, Verwandte) weitergegeben (mit Fremden klatscht man nicht über Freunde). Vertrauen wird insofern nur bedingt gebrochen - und gleichzeitig neu geschaffen -, die Vertraulichkeit bleibt in gewisser Weise auch insofern gewahrt, als extreme Formulierungen vermieden bzw. durch nachfolgende positive Äußerungen relativiert werden. Eine aus dem sozialen Beziehungsgeflecht ableitbare Verpflichtung zur ,Solidarität" mit dem abwesenden Klatschobjekt - die nicht zuletzt schon in der Tatsache, daß man morgen schon über die Person, mit der man heute klatscht, klatschen kann ${ }^{16}$ - unter-

${ }^{16}$ Gerade dieser potentielle Wechsel gehört konstitutiv zu einem „Klatschnetz" oder einer „Klatschzelle“, wie Elisabeth Bott (1957) dies nennt. Vgl. dazu auch M. Gluckman (1963). scheidet den Klatsch nicht zuletzt von anderen Genres der mündlichen Kommunikation, wie zum Beispiel dem ,Gerücht' oder der ,Verleumdung', für die diese ,Selbstbeschränkung' in der Wahl der Evaluationen nicht (oder in anderer Weise) gilt. ${ }^{17}$

Nachdem wir nun die Art und Weise, wie geklatscht und auch den Inhalt, über den geklatscht wird, wenigstens ansatzweise bestimmen konnten, bleiben Ort und Zeit der Praktizierung dieser mündlichen Aktivität nach wie vor offen. Zum charakteristischen ,Fluidum' des Klatsches gehört gerade aber auch, daß er beiläufig, en passant und (scheinbar oder tatsächlich) ohne feste Absicht praktiziert wird. Geklatscht wird zu Hause, im Büro, im Gasthaus, im Wartezimmer, in der Kantine, beim Friseur, beim Einkauf etc. Klatsch begleitet häufig andere Tätigkeiten, findet parallel zu diesen, nach deren Beendigung oder in Unterbrechungen und Pausenzeiten statt. Die Geeignetheit des Ortes bemißt sich primär danach, ob es möglich ist, ein persönliches Gespräch dergestalt zu führen, daß unliebsame Zuhörer ausgeschlossen werden können. ${ }^{18}$ Findet Klatsch allerdings in einem ,anonymen" sozialen Milieu statt, so reicht häufig ein Senken der Stimme aus, um die gewünschte und notwendige Abschirmung zu sichern. ${ }^{19}$ Die Zeit zum Klatschen muß in aller Regel von einer anderen Tätigkeit abgezogen werden Arbeit, Einkauf -, diese setzt so aber auch den

${ }^{17}$ Gegen eine Gleichsetzung von Klatsch mit Gerücht spricht unter anderem ein Unterschied in der möglichen Nachprüfbarkeit des Wahrheitsgehalts: Wer ein Gerücht in die Welt setzt, beruft sich im Zweifelsfall auf die Richtigkeit seiner Informationen; wer des Klatschens bezichtigt wird, beruft sich auf die Legitimität der Weitergabe von Informationen. Zudem wird Klatsch nur selten einer Nachprüfung unterzogen, denn diese verletzt sein Geheimnis und die Vertraulichkeit, mit der die Informationen weitergegeben wurden. Zudem ist es allein schon kompromittierend, wenn man zugibt, an einem Klatschgespräch beteiligt gewesen zu sein.

${ }^{18} \mathrm{Vgl}$. z. B. das Schließen einer sonst offenen Türe, eine räumliche Abgrenzung, die Bildung eines Grüppchens etc.

${ }^{19}$ Dies verwundert kaum, wenn man bedenkt, daß Klatsch zum einen hochindexikalisch und damit nur für jemanden verständlich ist, der die entsprechenden Hintergründe kennt, und daß zum anderen eine Klatschgeschichte nur für denjenigen interessant und von sozialer Relevanz ist, der das Klatschobjekt kennt. 
Rahmen, in dem sich Klatschgespräche abspielen. Eine eher eng begrenzte zeitliche Dauer ist für die Mehrzahl der Klatschgespräche, die häufig in Form eines ,laufenden Kommentars' zu aktuellen und/oder vergangenen Ereignissen produziert werden, typisch, im Unterschied zu ,ausgiebigen Klatschexzessen, in denen nichts anderes getan wird, als Bekannte und Freunde im Hinblick auf klatschträchtige Informationen durchzugehen (vgl. dem Klatsch verwandte Formen wie ,Durchhecheln' und Ähnliches). Gleichwohl kann auch die Zeitdauer eines ,normalen' Klatschgesprächs erheblichen Schwankungen unterliegen. Ein Merkmal der Klatschkommunikation ist gerade, daß sie sowohl relativ ausführlich, als auch stark verdichtet realisiert werden kann. Dies betrifft zum einen die Klatschpräsequenz - wie wir bereits gesehen haben -, es betrifft aber auch die Klatschgeschichte. Handelt es sich zum Beispiel um eine Erzählung über jemanden, über den noch nie geklatscht wurde, so bedarf die Etablierung eines individuellen moralischen Charakters einer ausführlicheren, detaillierteren und häufig aus mehreren Episoden zusammengesetzten Darstellung (so z. B. in „Ganz Umgänglich"). Ist der Protagonist aber demgegenüber schon eingeführt und sind dessen spezifische (klatschträchtige und klatschwürdige) Eigenschaften schon zumindest ansatzweise bekannt, so kann ein neues Ereignis, das eine derart bereits bekannte Eigenschaft in einem bis dahin vielleicht unbekannten oder unvermuteten Ausmaß vor Augen führt, diese Eigenschaft erneut klatschwürdig machen. Eine solche Klatschgeschichte kann aus einem einzigen Satz, aus einer kurzen Mitteilung bestehen, deren ,Klatsch-Trächtigkeit' durch die Intonation (begleitender Tonfall der Entrüstung oder des Lachens, Modulation der Stimme, Senken der Lautstärke) oder durch nonverbales Ausdrucksverhalten (hochgezogene Augenbrauen, geschürzte Lippen, Schütteln des Kopfes, indignierter Gesichtsausdruck) deutlich gemacht wird. $\mathrm{Zu}$ den Realisierungsbedingungen des Klatsches gehört auch, daß er jederzeit unter- oder abgebrochen werden kann, wenn die situativen Umstände dies erforderlich machen. Das Eintreffen des Familienmitglieds, über das gerade geredet wurde, oder das Auftauchen des Chefs, dessen Handlungsweise in der Arbeitspause diskutiert wird, kann ein Klatschgespräch ebenso abrupt zum Verstummen bringen wie ein Ereignis der Außenwelt, das die Aufmerksamkeit eines (oder mehrerer) Gesprächspartner mehr fesselt. ${ }^{20}$

Klatsch, so läßt sich abschließend festhalten, besteht aus Komponenten des Rekonstruierens, Inter- pretierens und Urteilens, die in dieser Reihenfolge, aber mit unterschiedlicher Gewichtung seinen Ablauf bestimmen. Sie schaffen zusammen erst den für den Klatsch charakteristischen Rhythmus der Erkundung, der Geschichten kreiert und das Leben in Geschichte verwandelt. Klatsch, so zeigt ein Blick auf seine Morphologie, unterscheidet sich nicht nur durch seinen Inhalt von anderen Formen der mündlichen Kommunikation; stilistische und ästhetische Elemente verweisen auch auf die zur Ausübung dieses Genres notwendige ,Kunstfertigkeit' gelungenen Klatschens. Für den Klatsch, dies sollte gezeigt werden, lassen sich konstitutive Merkmale extrapolieren. Im Klatsch wird außergewöhnliches Verhalten beschrieben und kommentiert: Klatschgeschichten rekurrieren auf tatsächliches, beobachtetes oder miterlebtes Geschehen, die spezifische personale Konstellation, die den Klatsch erst ermöglicht, findet ihren Ausdruck in einer gewissen Distanz des Erzählers zum Erzählten, schlägt sich in der Wahl evaluativer Formulierungen nieder und sorgt überdies für eine Begrenzung der Informationsdistribution. Die Interpretation des Geschehens, in der auf soziale Generalisierungen und Typisierungen zurückgegriffen wird, dient einer wechselseitigen Versicherung sozialer und moralischer Wertvorstellungen und Konventionen. Geklatscht wird - und dies ist, wie nicht vergessen werden sollte, nicht zuletzt eines seiner wichtigsten Merkmale - zum Vergnügen, zur Unterhaltung. Klatsch fördert das Gespräch, schafft Vertrauen und dient der Geselligkeit, die, wie Simmel $\left({ }^{4} 1984: 64\right)$ schreibt, „die Spielform auch für die ethischen Kräfte der konkreten Gesellschaft ist."

\section{Literatur}

Barthes, R., 1985: Fragmente einer Sprache der Liebe Frankfurt: Suhrkamp.

Bergmann, J. R., 1985: Zur Soziologie alltäglicher Interaktion - am Beispiel Klatsch. Manuskript Konstanz.

Bergmann, J. R., 1986: Klatsch als Gattung der alltäglichen Kommunikation. Zur Sozialform der diskreten Indiskretion. Habilitationsschrift Konstanz.

${ }^{20}$ Ein Klatschgespräch, das durch irgendein Ereignis unterbrochen wird, wird nicht in jedem Fall fortgesetzt, weder zum aktuellen, noch zu einem späteren Zeitpunkt; es kann aber wieder aufgenommen werden, sowohl direkt nachdem ein ,störendes Ereignis behoben ist als auch zu einem späteren Zeitpunkt (mit oder ohne Rekurs auf das abgebrochene Gespräch). 
Bott, E., 1957: Family and social network. London: Tavistock.

Gluckman, M., 1963: Gossip and scandal. Current Anthropology $3: 307-316$.

Goethe, J. W. v., ${ }^{8} 1973$ : Unterhaltungen deutscher Ausgewanderten. S. 125-241, in: Goethe, Werke, hrsg. von E. Treuz, München: Beck.

Harding, S., 1975: Women and words in a spanish village. S. 283-308, in: R. R. Reiter (ed.), Toward an anthropology of women. New York/London: Monthly Review Press.

Haviland, J. B., 1977: Gossip, reputation and knowledge in Zinacantan. Chicago: University Press.

Heidegger, M., 1979: Sein und Zeit. 15. Auflage, Tübingen: Niemeyer.

Jefferson, G., 1978: Sequential aspects of storytelling in conversation. S. 219-248, in: J. Schenkein (ed.), Studies in the organisation of conversational interaction. New York: University Press.

Kierkegaard, S., 1959: Der Augenblick. Aufsätze und Schriften des letzten Streits. Düsseldorf/Köln: Diederichs.
Paine, R., 1967: What is gossip about? An alternative hypothesis. Man (ns) $2:$ 278-285.

Pomerantz, A., 1975: Second assessments: A study of some features of agreements/disagreements. Manuskript University of California: Irvine.

Sabini, J./M. Silver, 1982: Moralities of everyday life. Oxford: Univ. Press.

Schegloff, E./H. Sacks, 1973: Opening up closings. Semiotica 8 : 289-327.

Schütz, A.Th. Luckmann, 1979: Strukturen der Lebenswelt. Frankfurt: Suhrkamp.

Simmel, G., 1984: Grundfragen der Soziologie. Berlin: de Gruyter.

Suls, J. M., 1977: Gossip as social comparison. Journal of Communication $27:$ 145-170.

Terasaki, A., 1976: Pre-announcement sequences in conversation. Social Science Working Paper. University of California : Irvine. 